\title{
Faster Approximation for Maximum Independent Set on Unit Disk Graph
}

\author{
Subhas C. Nandy, Supantha Pandit*, Sasanka Roy \\ Indian Statistical Institute, Kolkata, India
}

\begin{abstract}
Maximum independent set from a given set $D$ of unit disks intersecting a horizontal line can be solved in $O\left(n^{2}\right)$ time and $O\left(n^{2}\right)$ space. As a corollary, we design a factor 2 approximation algorithm for the maximum independent set problem on unit disk graph which takes both time and space of $O\left(n^{2}\right)$. The best known factor 2 approximation algorithm for this problem runs in $O\left(n^{2} \log n\right)$ time and takes $O\left(n^{2}\right)$ space [1, 2].

Keywords: Maximum independent set, Unit disk graph, Approximation algorithm.
\end{abstract}

\section{Introduction}

Intersection graphs of geometric objects have used to model several problems that arise in real scenarios [3]. Two important applications of these graphs are frequency assignment in cellular networks $[4,5]$ and map labeling [6]. If the geometric objects are disks then the corresponding intersection graph $G(V, E)$ is called the disk graph. Here the vertex set $V$ corresponds to a given set of disks in the plane, and there is an edge between two vertices in $V$ iff the corresponding two disks intersect.

A unit disk graph is an intersection graph where each disk is of diameter 1. Let $G(V, E)$ be a given graph. A set $V^{\prime} \subseteq V$ is said to be an independent set of $G$ if no two vertices in $V^{\prime}$ are connected by an edge in $G$. In the maximum

\footnotetext{
*Corresponding author

Email addresses: nandysc@isical.ac.in (Subhas C. Nandy), pantha.pandit@gmail.com (Supantha Pandit), sasanka@isical.ac.in (Sasanka Roy)
} 
independent set (MIS) the goal is to find an independent set $V^{\prime}$ which has the maximum cardinality. In this paper, we consider the following problem.

Maximum Independent Set on Unit Disk Graph (MISUDG): Given a unit disk graph $G(V, E)$, find an independent set of $G$ whose cardinality is maximum.

To provide an approximation algorithm for $M I S U D G$, we consider the following problem.

MISUDG-L: Given a set $D_{i}$ of $n_{i}$ unit disks that are intersected by horizontal line $L_{i}$, find a subset $D^{\prime} \subseteq D_{i}$ of maximum cardinality such that no two disks in $D^{\prime}$ have a common intersection point.

Related Work: The MISUDG problem is known to be NP-complete [7, 8, 9]. In Table 1, we demonstrate a comparison study of the progress on MISUDG.

\begin{tabular}{|l|c|c|c|}
\hline Reference & Factor & Time & Space \\
\hline Marathe et al. [10] & 3 & $O\left(n^{2}\right)$ & $O(n)$ \\
\hline Das et al. [11] & 2 & $O\left(n^{3}\right)$ & $O\left(n^{2}\right)$ \\
\hline Jallu and Das [1] & 2 & $O\left(n^{2} \log n\right)$ & $O\left(n^{2}\right)$ \\
\hline Das et al. [2] & 2.16 & $O\left(n \log ^{2} n\right)$ & $O(n \log n)$ \\
\hline Theorem 5 & 2 & $O\left(n^{2}\right)$ & $O\left(n^{2}\right)$ \\
\hline
\end{tabular}

Table 1: Comparison table

Matsui [12] consider the MISUDG problem. If the disk centers are located inside a strip of fixed height $k$, then this problem can be solved in $O\left(n^{4\left\lceil\frac{2 k}{\sqrt{3}}\right\rceil}\right)$ time. Further, for any integer $r \geq 2$, Matsui [12] provided a $\left(1-\frac{1}{r}\right)$ factor approximation algorithm for the same problem which takes $O\left(r n^{4\left\lceil\frac{2(r-1)}{\sqrt{3}}\right\rceil}\right)$ time and $O\left(n^{2 r}\right)$ space. Das et al. [11], also designed a PTAS for MISUDG problem by using the shifting strategy of Hochbaum and Maass [13]. For a given positive integer $k>1$, they gave a $\left(1+\frac{1}{k}\right)^{2}$ factor approximation algorithm which runs in $O\left(k^{4} n^{\sigma_{k} \log k}+n \log n\right)$ time and $O(n+k \log k)$ space, where $\sigma_{k} \leq \frac{7 k}{3}+2$. Recently, Jallu and Das [1], improved the running time of the same problem to 
$n^{O(k)}$ by keeping the approximation factor same. A fixed parameter tractable algorithm for the MISUDG problem was proposed by van Leeuwen [14]. The running time of that algorithm is $O\left(t^{2} 2^{2 t} n\right)$, where the parameter $t$ represents the thickness ${ }^{1}$ of the $U D G$.

\section{Our Contributions:}

- We design an exact algorithm for $M I S U D G-L$ problem which runs in $O\left(n^{2}\right)$ time using $O\left(n^{2}\right)$ space.

- We design a factor 2 approximation algorithm for $M I S U D G$ problem which takes both $O\left(n^{2}\right)$ time and space. It is an improvement over the best known result on this problem proposed by Jallu et al. [1]. They gave a factor 2 approximation algorithm for this problem where the time and space complexities are $O\left(n^{2} \log n\right)$ and $O\left(n^{2}\right)$ respectively.

Notations and Definitions: Let $D=\left\{d_{1}, d_{2}, \ldots, d_{n}\right\}$ be a set of $n$ unit disks in the plane. The center of the disk $d_{i} \in D$ is $c_{i}$. The $x$-coordinate of $c_{i}$ is $x\left(c_{i}\right)$. For a given set $S$ of disks, $|S|$ is the cardinality of $S$. The line segment connecting two points $s$ and $t$ is denoted by $\overline{s t}$.

\section{2. $O\left(n^{2}\right)$ time exact algorithm for $M I S U D G-L$ problem}

In this section, we design an exact dynamic programming based algorithm for $M I S U D G-L$ problem. Let $D_{i}=\left\{d_{1}, d_{2}, \ldots d_{n_{i}}\right\}$ be a set of $n_{i}$ unit disks intersecting a horizontal line $L_{i}$. We partition the set $D_{i}$ into two sets $D_{i}^{a}$ and $D_{i}^{b}$, where $D_{i}^{a}$ is the set of all disks in $D_{i}$ whose centers are above the horizontal line $L_{i}$ and $D_{i}^{b}$ is the set of all disks in $D_{i}$ whose centers are below the horizontal line $L_{i}$. To design the dynamic programming algorithm, we need the following two lemmas.

Lemma 1. Let $d_{1}, d_{2}, d_{3} \in D_{i}^{a}$ be three disks with centers $c_{1}, c_{2}$, and $c_{3}$ respectively. Assume that $x\left(c_{1}\right)<x\left(c_{2}\right)<x\left(c_{3}\right)$. Now if $d_{1}, d_{2}$ and $d_{2}, d_{3}$ are non-intersecting, then $d_{1}, d_{3}$ are non-intersecting.

\footnotetext{
${ }^{1} \mathrm{~A} U D G$ is said to have thickness $t$, if each strip in the slab decomposition of width 1 of the $U D G$ contains at most $t$ disk centers
} 
Proof. Suppose on the contrary, we assume that $d_{1}$ and $d_{3}$ are intersecting. Then clearly the line segment $\overline{c_{1} c_{3}}$ must be fully covered by $d_{1}$ and $d_{3}$. Since $x\left(c_{1}\right)<x\left(c_{2}\right)<x\left(c_{3}\right), c_{2}$ can not be above $\overline{c_{1} c_{3}}$. Otherwise, it must intersect $\overline{c_{1} c_{3}}$ and hence intersect either $d_{1}$ or $d_{2}$. Further, the perpendicular distance between the horizontal line $L_{i}$ and any point on $\overline{c_{1} c_{3}}$ is at most 1 . Then, if $c_{2}$ is

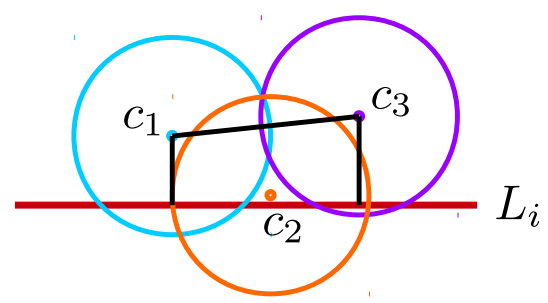

Figure 1: Proof of Lemma 1.

60

below $\overline{c_{1} c_{3}}$, it must intersect $\overline{c_{1} c_{3}}$ as the centers are above the horizontal line $L_{i}$. Therefore, we have arrived at a contradiction that either $d_{1}, d_{2}$ are intersecting or $d_{2}, d_{3}$ are intersecting.

Lemma 2. Let $d_{1}, d_{2} \in D_{i}^{b}$ and $d_{3} \in D_{i}^{a}$ be three disks with centers $c_{1}, c_{2}$, and $c_{3}$ respectively. Assume that $x\left(c_{1}\right)<x\left(c_{2}\right)<x\left(c_{3}\right)$. Now if $d_{1}, d_{2}$ and $d_{2}, d_{3}$ are non-intersecting, then $d_{1}, d_{3}$ are non-intersecting.

Proof. Suppose on the contrary, we assume that $d_{1}$ and $d_{3}$ are intersecting. Then clearly $\overline{c_{1} c_{3}}$ is at most 1 . Also by the assumption, both $\overline{c_{1} c_{2}}$ and $\overline{c_{2} c_{3}}$ are greater than 1 . Let $V_{L}$ be a vertical line through $c_{2}$ (see Figure 2). The 70 two lines $L_{i}$ and $V_{L}$ intersect at a point $O$ and partition the space into four quadrants: ' ++ ', ' +- ', ' -- ', and ' -+ '. The point $c_{3}$ is in ' ++ ', whereas $c_{1}$ is in '--'. Now consider an unit disk $d^{*}$ whose center coincides with $O$. Note that, all disks in $D_{i}$ intersect the line $L_{i}$. Hence the disk $d_{2}$ contains the point $O$. Further, since $c_{2}$ and $c_{3}$ are non-intersecting, $c_{3}$ must be outside $d^{*}$.

Take the segment $\overline{c_{2} c_{3}}$ which intersect $d^{*}$ at $c_{3}^{\prime}$. Further, extend the segment $\overline{c_{2} c_{3}}$ in the direction of $c_{2}$ such that it intersect another point $c_{2}^{\prime}$ on $d^{*}$. Consider the segment $\overline{c_{2}^{\prime} c_{3}^{\prime}}$. Now by an easy observation, we say that, the voronoi partition line ( $V P L)$ of $c_{2}^{\prime}$ and $c_{3}^{\prime}$ passes through $O$ and intersects the two quadrants ' +- ' and ' -+ '. Again, consider the segment $\overline{c_{2} c_{3}^{\prime}}$. Since $c_{2}$ is on the line through $c_{2}^{\prime}$ 


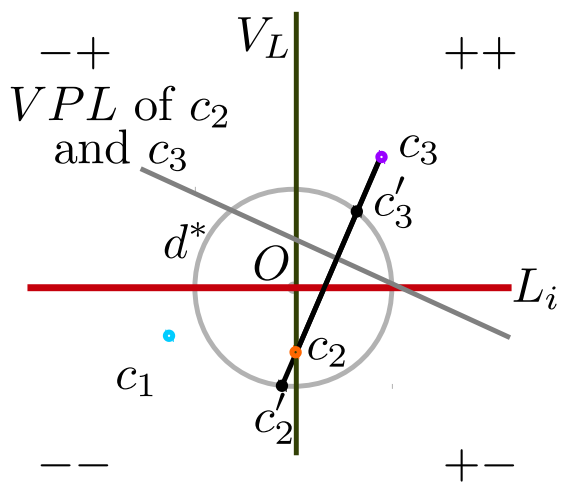

Figure 2: Proof of Lemma 2

so and $c_{3}^{\prime}$, the slope of the $V P L$ of $c_{2}$ and $c_{3}^{\prime}$ must be the same as that of $c_{2}^{\prime}$ and $c_{3}^{\prime}$. Further, this $V P L$ is to the right of the $V P L$ of $c_{2}^{\prime}$ and $c_{3}^{\prime}$ and contains the whole '--' quadrant to its left. Due to similar argument, the $V P L$ of $c_{2}, c_{3}$ contains the whole '--' quadrant to its left. Since $c_{1}$ and $c_{2}$ are in '--' quadrant, clearly the point $c_{1}$ is closer to $c_{2}$ than $c_{3}$. Therefore, $\overline{c_{1} c_{3}}$ is greater than 1 , since $\overline{c_{1} c_{2}}$ is greater than 1 . This leads to the contradiction that $\overline{c_{1} c_{3}}$ is at most 1.

We now describe the algorithm as follows. Let $\left\{d_{1}^{a}, d_{2}^{a}, \ldots, d_{n_{1}}^{a}\right\}$ be the set of disks in $D_{i}^{a}$ sorted according to their increasing $x$-coordinates. Similarly, let $\left\{d_{1}^{b}, d_{2}^{b}, \ldots, d_{n_{2}}^{b}\right\}$ be the set of disks in $D_{i}^{b}$ sorted according to their increasing эo $x$-coordinates. We add two new disks $d_{0}^{a}$ and $d_{0}^{b}$ which satisfies the following, (i) $d_{0}^{a}$ is to the left of $d_{1}^{a}$ and $d_{0}^{b}$ is to the left of $d_{1}^{b}$, (ii) both $d_{0}^{a}$ and $d_{0}^{b}$ are independent with the disks in $D_{i}$, and (iii) $d_{0}^{a}$ and $d_{0}^{b}$ do not intersect each other. For any disk $d \in D_{i}\left(d \neq\left\{d_{0}^{a}, d_{0}^{b}\right\}\right)$, define $R I^{a}(d)$ (resp. $\left.R I^{b}(d)\right)$ be the rightmost disk in $D_{i}^{a}$ (resp. $D_{i}^{b}$ ) which is independent with $d$ and whose center is to the left of the center of $d$.

We define a subproblem $S(k, \ell)$, for $0 \leq k \leq n_{1}$ and $0 \leq \ell \leq n_{2}$, to be the set of all disks in $D_{i}^{a}$ which are to the left of the disk $d_{k}^{a} \in D_{i}^{a}$ and set of all disks in $D_{i}^{b}$ which are to the left of the disk $d_{\ell}^{b} \in D_{i}^{b}$. Let $I(k, \ell)$ be an optimal set of independent unit disks in $S(k, \ell)$, and let $V(k, \ell)$ be the value of this solution.

100 Lemma 3. Let $D_{i}^{a}(k)=\left\{d_{1}^{a}, d_{2}^{a}, \ldots, d_{k}^{a}\right\}$ be a set of $k$ leftmost disks in $D_{i}^{a}$ and $D_{i}^{b}(\ell)=\left\{d_{1}^{b}, d_{2}^{b}, \ldots, d_{\ell}^{b}\right\}$ be the set of $\ell$ leftmost disks in $D_{i}^{b}$. Now, 
A. if $x\left(c_{k}^{a}\right)>x\left(c_{\ell}^{b}\right)$, then

(1) if $d_{k}^{a} \in I(k, \ell)$, then $V(k, \ell)=V\left(R I^{a}\left(d_{k}^{a}\right), R I^{b}\left(d_{k}^{a}\right)\right)+1$

(2) if $d_{k}^{a} \notin I(k, \ell)$, then $V(k, \ell)=V(k-1, \ell)$

B. if $x\left(c_{k}^{a}\right)<x\left(c_{\ell}^{b}\right)$, then

(3) if $d_{\ell}^{b} \in I(k, \ell)$, then $V(k, \ell)=V\left(R I^{a}\left(d_{\ell}^{b}\right), R I^{b}\left(d_{\ell}^{b}\right)\right)+1$

(4) if $d_{\ell}^{b} \notin I(k, \ell)$, then $V(k, \ell)=V(k, \ell-1)$

Proof. We prove cases 1 and 2. The proof of the cases 3 and 4 are similar. Here we assume that, $x\left(c_{k}^{a}\right)>x\left(c_{\ell}^{b}\right)$, i.e., the disk $d_{k}^{a}$ is to the right of the disk $d_{\ell}^{b}$. Let $T^{*}$ be a maximum independent set of disks for subproblem $S(k, \ell)$. There are two possibilities, either $d_{k}^{a}$ is in the optimal solution or not.

$d_{k}^{a} \in I(k, \ell)$ : Let us assume that, $d_{\tau}^{a}=R I^{a}\left(d_{k}^{a}\right)$ and $d_{\nu}^{b}=R I^{b}\left(d_{k}^{a}\right)$. Since, $d_{k}^{a}$ is in the optimal solution, no disk in $D_{i}^{a}\left(\right.$ resp. $\left.D_{i}^{b}\right)$ whose center is in between the centers of $d_{\tau}^{a}$ (resp. $\left.d_{\nu}^{b}\right)$ and $d_{k}^{a}$ can be present in any feasible solution. Thus any feasible solution contains disks from $D_{i}^{a}(\tau)$ and $D_{i}^{b}(\nu)$. Therefore, $T^{*}$ consists of $d_{k}^{a}$, together with the optimal solution to the subproblem $S(\tau, \nu)$.

$d_{k}^{a} \notin I(k, \ell)$ : By an argument similar to case 1 , we say that, an optimal solution for $D_{i}^{a}(k-1)$ and $D_{i}^{b}(\ell)$ gives an optimal solution for $D_{i}^{a}$ and $D_{i}^{b}$.

120 This completes the proof of the lemma.

Therefore, Lemma 3 suggests the following recurrence relation:

$$
V(k, \ell)=\max \left\{\begin{array}{l}
V\left(R I^{a}\left(d_{k}^{a}\right), R I^{b}\left(d_{k}^{a}\right)\right)+1, \\
V(k-1, \ell), \\
V\left(R I^{a}\left(d_{\ell}^{b}\right), R I^{b}\left(d_{\ell}^{b}\right)\right)+1, \\
V(k, \ell-1),
\end{array}\right\} \text { for } x\left(c_{k}^{a}\right)>x\left(c_{\ell}^{b}\right)
$$

Optimal Solution: The optimal solution can be found by calling the function $V\left(n_{1}, n_{2}\right)$ with the base cases $V(k, \ell)=0$ where both $k, \ell=2$. Clearly, the final optimal solution contains the disks $d_{0}^{a}$ and $d_{0}^{b}$. Hence, we reduce the value of the optimal solution by 2 and remove these two disks from the optimal solution. 
Running time: Let $T\left(n_{i}\right)$ be the total time taken by an algorithm $\mathcal{Z}$ to evaluate $V\left(n_{1}, n_{2}\right)$. For a particular disk $d \in D_{i}$, finding either $R I^{a}(d)$ or $R I^{b}(d)$ requires $O\left(n_{i}\right)$ time. Hence, in $O\left(n_{i}^{2}\right)$ time, we find $R I^{a}(d)$ and $R I^{b}(d)$ for all $d \in D_{i}$. During recursive calls, for a particular disk $d$, the disks $R I^{a}(d)$ and $R I^{b}(d)$ can be found in $O(1)$ time. Therefore, the running time of $\mathcal{Z}$ will be $O\left(n_{i}^{2}\right)$. Further, this algorithm requires $O\left(n_{i}^{2}\right)$ space to store the values of $V(k, \ell)$, for $0 \leq k \leq n_{1}$ and $0 \leq \ell \leq n_{2}$. Finally, we now have the following theorem.

Theorem 4. MISUDG-L problem can be solved optimally in $O\left(n_{i}^{2}\right)$ time and $O\left(n_{i}^{2}\right)$ space.

\section{3. $O\left(n^{2}\right)$ time factor 2 approximation for MISUDG problem}

In this section, we design a factor 2 approximation algorithm for MISUDG problem. Let $D=\left\{d_{1}, d_{2}, \ldots, d_{n}\right\}$ be a set of $n$ unit disks in the plane. We first place horizontal lines from top to bottom with unit distance between each consecutive pair. Assume that there are $k$ such horizontal lines $\left\{L_{1}, L_{2}, \ldots, L_{k}\right\}$. Let $D_{i} \subseteq D$ be the set of disks which are intersected by the line $L_{i}$. Now we have the following observation.

Observation 1. Any two disks, $d \in D_{i}$ and $d^{\prime} \in D_{j}$ are independent (nonintersecting) if $|i-j|>1$, for $1 \leq i, j \leq k$.

Note that, algorithm $\mathcal{Z}$ optimally solves $M I S U D G-L$ problem. Run $\mathcal{Z}$ on each $D_{i}$, for $1 \leq i \leq k$ and let $S_{i}$ be an independent set of unit disks of maximum cardinality in $D_{i}, 1 \leq i \leq k$. Let $S_{\text {odd }}=\bigcup_{\substack{1 \leq i \leq k \\ i \text { is odd }}}, S_{i}$ and $S_{\text {even }}=\bigcup_{\substack{1 \leq i \leq k \\ i \text { is even }}}, S_{i}$. We set $S$ as $S_{\text {odd }}$ or $S_{\text {even }}$ depending on whether $\left|S_{\text {odd }}\right|$ is greater or less than $\left|S_{\text {even }}\right|$ and report $S$ as the result of our algorithm. We now have the following theorem.

Theorem 5. The time and space complexities of our algorithm are both $O\left(n^{2}\right)$ and it produces a result with approximation factor 2 .

Proof. Let Opt be an optimal solution for $D$. Form Observation 1, we say that the disks in $S_{\text {odd }}$ are independent, and so $S_{\text {even }}$. Also, we have $\left|S_{\text {odd }}\right|+\left|S_{\text {even }}\right| \geq$ $\mid$ Opт $\mid$. Therefore, $2|S|=2 \max \left\{\left|S_{\text {odd }}\right|,\left|S_{\text {even }}\right|\right\} \geq\left|S_{\text {odd }}\right|+\left|S_{\text {even }}\right| \geq \mid$ OpT $\mid$. 
Since disks in $S_{\text {odd }}$ and $S_{\text {even }}$ are mutually independent, the total time required for computing $S_{\text {odd }}$ or $S_{\text {even }}$ is $O\left(n^{2}\right)$. Hence, the total time for reporting $S$ is $O\left(n^{2}\right)$, as required. For each $D_{i}, \mathcal{Z}$ takes $O\left(n^{2}\right)$ space. Hence, the total space complexity is $O\left(n^{2}\right)$.

\section{References}

[1] R. K. Jallu, G. K. Das, Improved algorithm for maximum independent set on unit disk graph, in: CALDAM, 2016, pp. 212-223.

[2] G. K. Das, G. D. da Fonseca, R. K. Jallu, Efficient independent set approximation in unit disk graphs, unpublished Manuscript (2016).

[3] F. Roberts, Graph Theory and Its Applications to Problems of Society, Society for Industrial and Applied Mathematics, 1978.

[4] W. K. Hale, Frequency assignment: Theory and applications, Proceedings of the IEEE 68 (12) (1980) 1497-1514.

[5] E. Malesińska, Graph-theoretical models for frequency assignment problems, Ph.D. thesis, Technische Universität Berlin, Berlin, Germany. (1997).

[6] P. K. Agarwal, M. van Kreveld, S. Suri, Label placement by maximum independent set in rectangles, Compu. Geom. 11 (3) (1998) $209-218$.

[7] D. Wang, Y.-S. Kuo, A study on two geometric location problems, Information Processing Letters 28 (6) (1988) $281-286$.

[8] M. R. Garey, D. S. Johnson, Computers and Intractability: A Guide to the Theory of NP-Completeness, W. H. Freeman \& Co., NY, USA, 1979.

[9] B. N. Clark, C. J. Colbourn, D. S. Johnson, Unit disk graphs, Discrete Mathematics 86 (1) (1990) 165 - 177.

[10] M. V. Marathe, H. Breu, H. B. Hunt, S. S. Ravi, D. J. Rosenkrantz, Simple heuristics for unit disk graphs, Networks 25 (2) (1995) 59-68.

[11] G. K. Das, M. De, S. Kolay, S. C. Nandy, S. Sur-Kolay, Approximation algorithms for maximum independent set of a unit disk graph, Information Processing Letters 115 (3) (2015) 439 - 446. 
[12] T. Matsui, Approximation algorithms for maximum independent set problems and fractional coloring problems on unit disk graphs, in: JCDCG, 1998, pp. 194-200.

[13] D. S. Hochbaum, W. Maass, Approximation schemes for covering and packing problems in image processing and VLSI, J. ACM 32 (1) (1985) $130-$ 136.

190 [14] E. J. van Leeuwen, Approximation algorithms for unit disk graphs, in: WG, 2005, pp. 351-361. 